\title{
$t^{4}$ Workshop Report*
}

\section{A Framework Program for the Teaching of Alternative Methods (Replacement, Reduction, Refinement) to Animal Experimentation}

\author{
Mardas Daneshian ${ }^{1}$, Mohammad A. Akbarsha ${ }^{2}$, Bas Blaauboer ${ }^{3}$, Francesca Caloni ${ }^{4}$, \\ Pierre Cosson ${ }^{5}$, Rodger Curren ${ }^{6}$, Alan Goldberg ${ }^{7}$, Franz Gruber ${ }^{8}$, Frauke Ohl ${ }^{9}$, Walter Pfaller ${ }^{10}$, \\ Jan van der Valk ${ }^{11}$, Pilar Vinardell ${ }^{12}$, Joanne Zurlo ${ }^{7}$, Thomas Hartung ${ }^{1,7,13}$, and Marcel Leist ${ }^{1,14}$ \\ ${ }^{1}$ Center for Alternatives to Animal Testing - Europe, University of Konstanz, Germany; ${ }^{2}$ Mahatma Gandhi-Doerenkamp Center \\ (MGDC) for Alternatives to Use of Animals in Life Science Education, Bharathidasan University, Tiruchirappalli, India; ${ }^{3}$ Doerenkamp- \\ Zbinden Chair on Alternatives to Animal Testing in Toxicological Risk Assessment, Institute for Risk Assessment Sciences, Division \\ of Toxicology, Utrecht University, The Netherlands; ${ }^{4}$ Department of Veterinary Sciences Technologies for Food Safety, Università degli \\ Studi di Milano, Italy; ${ }^{5}$ Doerenkamp-Naef-Zbinden Chair on In vitro alternatives to animal experiments, Geneva Faculty of Medicine, \\ Switzerland; ${ }^{6}$ Institute for In Vitro Sciences, Inc., USA; ${ }^{7}$ Doerenkamp-Zbinden Chair for Evidence-based Toxicology and Center for \\ Alternatives to Animal Testing, Johns Hopkins Bloomberg School of Public Health, Dept. Environmental Health Sciences, Baltimore, \\ USA $;{ }^{8}$ Doerenkamp-Zbinden Foundation, Switzerland; ${ }^{9}$ Animal Welfare \& Laboratory Animal Science and Animals in Science \& \\ Society, Faculty of Veterinary Medicine, Utrecht University, The Netherlands; ${ }^{10}$ Innsbruck Medical University, Department \\ of Physiology and Medical Physics, Division Physiology, Austria; ${ }^{11}$ 3Rs-Centre Utrecht Life Sciences, and NKCA, Department of \\ Animals in Science and Society, Faculty of Veterinary Medicine, Utrecht University, The Netherlands; ${ }^{12}$ Departament de Fisiologia, \\ Facultat de Farmàcia, Universitat de Barcelona, Spain; ${ }^{13}$ University of Konstanz, Toxicology and Pharmacology, Konstanz, Germany; \\ ${ }^{14}$ Doerenkamp-Zbinden Chair for Biomedicine and in vitro Toxicology, University of Konstanz, Germany
}

\begin{abstract}
Summary
Development of improved communication and education strategies is important to make alternatives to the use of animals, and the broad range of applications of the $3 R$ s concept better known and understood by different audiences. For this purpose, the Center for Alternatives to Animal Testing in Europe (CAAT-Europe) together with the Transatlantic Think Tank for Toxicology $\left(t^{4}\right)$ hosted a three-day workshop on "Teaching Alternative Methods to Animal Experimentation".

A compilation of the recommendations by a group of international specialists in the field is summarized in this report. Initially, the workshop participants identified the different audience groups to be addressed and also the communication media that may be used. The main outcome of the workshop was a framework for a comprehensive educational program. The modular structure of the teaching program presented here allows adaptation to different audiences with their specific needs; different time schedules can be easily accommodated on this basis. The topics cover the 3Rs principle, basic research, toxicological applications, method development and validation, regulatory aspects, case studies and ethical aspects of $3 R$ s approaches. This expert consortium agreed to generating teaching materials covering all modules and providing them in an open access online repository.
\end{abstract}

Keywords: 3Rs, education, teaching, toxicology

\section{Introduction}

Background

The field of alternative methods to animal experimentation has been growing and diversifying during the last years, and it has reached out to many other disciplines. In this context, the term "alternative methods" is no longer precisely defining (Hartung et al., 2009). We therefore use the expression "3Rs methods" to refer to all approaches aiming at replacement, reduction or refinement of animal experiments. These include for instance integrated testing strategies, pathway-based omics, high-throughput and high-content testing, modeling and

${ }^{*}$ a report of $\mathrm{t}^{4}$ - the transatlantic think tank for toxicology, a collaboration of the toxicologically oriented chairs in Baltimore,

Konstanz and Utrecht sponsored by the Doerenkamp-Zbinden Foundation 


\section{Box 1}

For the introduction of modules and for assignment of exercise tasks, case studies were considered a helpful teaching tool. Some examples from different fields are given below.

\section{Examples for case studies:}

\section{Case study:}

Recombinant antibodies

Eye irritation

Thalidomide

Production of antibodies

Disease models (strategies)

Marine biotoxins

Pyrogen tests

Pregnancy tests (Xenopus)

Species differences

Infections

Bad experimental design

Aspirin

Phototoxicity

\section{Background and lesson to be learnt:}

The TeGenero trial showed that extensive safety testing in multiple animal species did not ensure human safety (TG1412).

In vitro models of a "cytokine storm" based on human cells are claimed to have higher predictively (Stebbings et al., 2007).

Different in vitro models have been developed and validated in the last years to replace the traditional Draize test in rabbits.

Validation studies of the alternatives suggested that the animal model is badly reproducible, and therefore makes any correlative efforts with alternative methods difficult.

A combination of several alternative test methods within a (tiered) testing strategy may be able to replace the in vivo Draize test, and several validation studies of different in vitro methods were undertaken or are on-going, e.g., organotypic in vitro assays (isolated eyes), cytotoxicity and cell-function based assays (e.g., red blood cell lysis), reconstructed human tissue (RhT)-based test methods, and quasi-in vivo methods such as the hen egg chorioallantoic membrane test.

Thalidomide, quite safe in adults, caused serious birth defects worldwide in the late 1950s and early 1960s. After this incident, the requirement of preclinical reproductive toxicity testing of drugs in Europe (EMEA) and the US (FDA) started. Additionally, species differences became strongly evident: the compound is hardly toxic to mice and rats, but only to New Zealand white rabbit.

Ascites and Freund's adjuvant were considered indispensable by all scientific experts not so long ago $\rightarrow$ now many substitutes exist. In vivo generation of monoclonal antibodies was thought to be unavoidable until very recently $\rightarrow$ now, pure in vitro selection (phage display) and cell culture production are emerging.

Non-mammalian models and induced pluripotent stem cells from patients with monogenetic and diseases have been used successfully for research and for drug discovery.

The mouse bioassay for marine biotoxin contaminations of seafood can now be replaced by polygenic chemical analytical methods for most of the toxins for which standards are available. The development of functional 3Rs methods in this area is ongoing.

Classical pyrogen testing uses large numbers of rabbits. The clotting reaction of Limulus haemolymph was the first alternative assay for Gram-negative pyrogens. The new generations of pyrogen tests based on the reaction of human immune cells (whole blood) covers the broad range of microbial and synthetic pyrogens relevant to human reactivity.

Pregnancy tests based on urine injection into frogs and observation of their egg production was state of the art until the 1960s. Antibody-based in vitro methods made such assays completely redundant.

Mouse livers are more prone to cancer (e.g., by peroxisome proliferators), than human or other species' livers; different types of tumors are often found in rodents and man; different brain size and development speed, e.g., postnatal day 5 in rat would still be in pregnancy in humans; different metabolites (e.g., citalopram is toxic to dogs but not to man); failure of stroke, Alzheimer's and Parkinson's Disease drugs in man that were successful in rodents; body size affects biokinetics and oxidative stress; different species may differ in (metabolic) enzymes, e.g., mice and rats synthesize vitamin C, humans and guinea pigs do not.

The case of virulence studies of infectious bacteria shows that each model has its own advantages and disadvantages. There is no golden standard, and quite surprisingly, the Dictyostelium discoideum amoebae model (Lima et al., 2011) has a high predictivity for distinction of clinically virulent strains from non-virulent strains.

Numerous examples can be found to illustrate the importance of experimental design and documentation. The ARRIVE guidelines give an example for minimal documentation requirements of animal experiments. Often, the attempt to save animals results in underpowered studies that then need to be repeated with more animals.

Acetylsalicylic acid is an irritant and potential toxicant and tumor promotor in animal models but is used safely in man.

The 3T3 NRU phototoxicity test was the first accepted alternative method in the OECD guidelines (TG 432). The 3T3 Neutral Red Uptake Phototoxicity Test, an in vitro assay with the Balb/c 3T3 mouse fibroblast cell line, is based on the comparison of cytotoxicity of a toxic substance in the presence or absence of UVA light. Cytotoxicity is evaluated as a concentration dependent reduction of the uptake of the vital dye Neutral Red. 
other in silico approaches, and systems biology, as far as they are aligned with the 3 Rs principles.

Because of their human relevance, and after extensive validation, several 3Rs methods in the area of toxicology have been adopted in regulatory guidance documents. The continuing progress in the field of in vitro methods, e.g., high-throughput and high-content imaging methods and advanced knowledge in the fields of systems biology, protein interactions and gene expression patterns (omics), have opened up new prospects, especially in the field of toxicology, to investigate pathway disturbances and human relevant targets. More generally, 3Rs methods are becoming an essential element in the whole field of biomedical research, from its most fundamental aspects to its daily applications. Today 3Rs methods represent a multidisciplinary scientific area comprising animal science, basic biology, test development, pharmacology, toxicology, regulations and regulatory practices, as well as ethics and behavioral sciences.

According to European Directive 2011/63/EU1 , all personnel working with experimental animals should be educated to be competent to work with animals. In many countries, courses have been established or are in the process of being established, to meet this requirement. Also other persons not directly involved in animal experiments but having responsibility in one way or another for animal experiments, should be educated and regularly updated on progress being made with respect to the $3 \mathrm{Rs}$ in order to maximize their implementation. These persons can be company managers, regulators and journalists inter alia.
Because of the complexity of this most diverse field it is mandatory that experts share their knowledge to develop comprehensive teaching programs.

\section{Workshop goals}

This workshop, held in Konstanz, Germany, was sponsored by the Doerenkamp-Zbinden-Foundation (DZF) to gather experts in teaching of different aspects of 3Rs methods. The objective was to provide a forum for the exchange of knowledge, definition of the status quo and design of a common strategy for improved knowledge dissemination to specific audiences in the field. Moreover, target groups and their specific educational needs were to be identified. A clear understanding of advantages and limitations of 3Rs approaches and animal models should be transmitted to all target groups (Box 1). The definition of lecture modules reflecting the state of the art of the different disciplines of 3Rs methods and 3Rs knowledge was the major task.

\section{Results overview}

\section{Overall teaching goals}

The aim of the dissemination of knowledge on 3Rs methods is to raise consciousness for the scientific soundness of the $3 \mathrm{Rs}$ methodology (Fig. 1). A better understanding of the ethical as well as the economic aspects of 3 Rs methods will provide a better judgment basis for weighing different approach options.

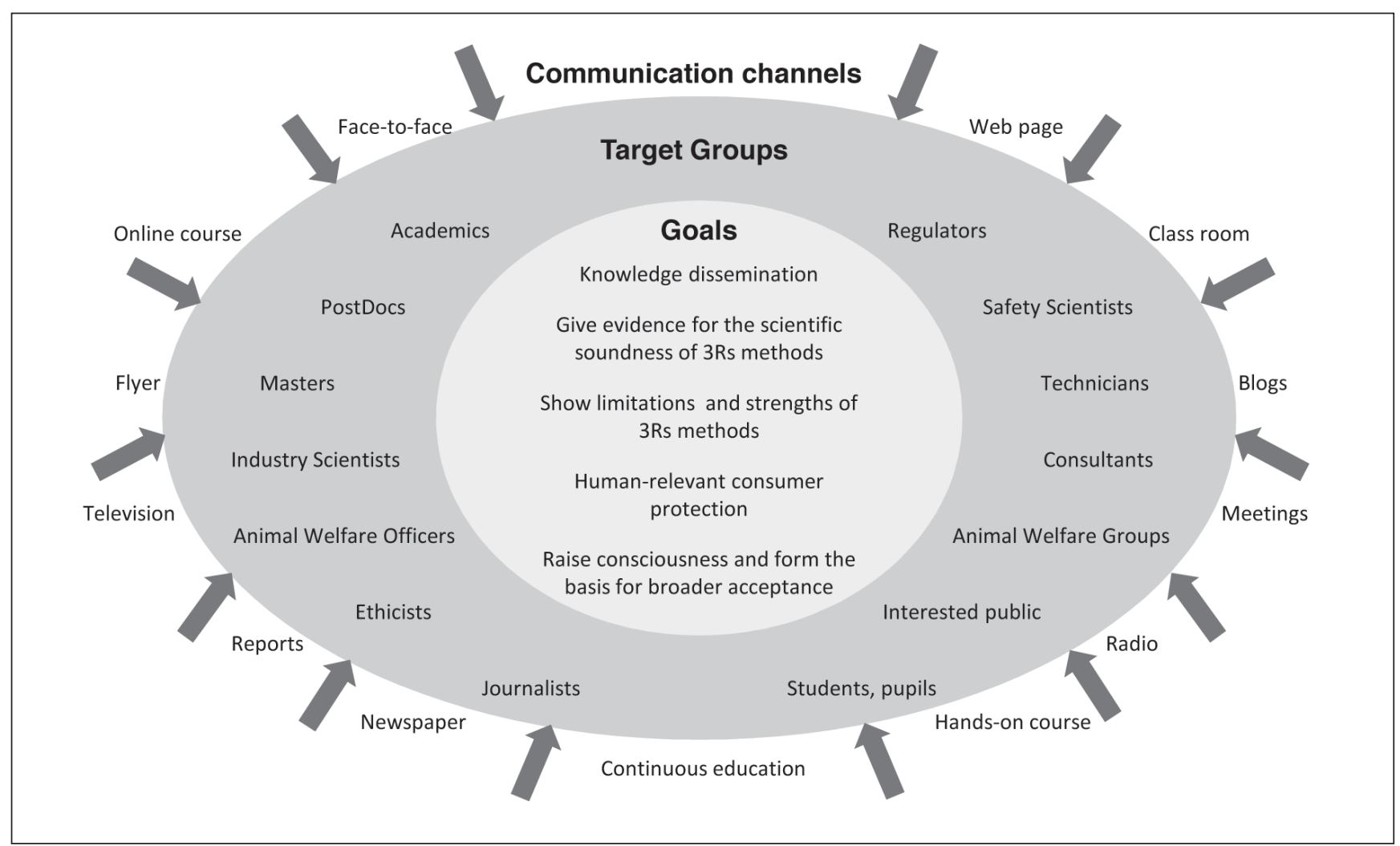

Fig. 1: Overview of the goals, target groups and communication channels

8 http://ec.europa.eu/environment/chemicals/lab_animals/pdf/sec_2010_1107.pdf 
Finally, an introduction to the new technological approaches in this rapidly developing field of biomedical research will foster an appreciation of the multidisciplinary and progressive nature of the field.

\section{Target groups}

Teaching 3Rs methods means covering the various multi-disciplinary aspects of this broad field for different audiences at the appropriate level of detail. The target audiences were classified into five overlapping general groups (Fig. 1):

a) Graduate scientists, e.g., PostDocs, PhDs, MScs, animal welfare officers

b) Regulators

c) Industry scientists, e.g., license holders, principal investigators, ethical committee members (IACUC and IRB), toxicologists (safety testing), pharmacologists, chemists, labtechnicians, regulatory officers, managers

d) Interested and educated public, e.g., journalists, animal welfare groups

e) Undergraduate students, pupils

As 3Rs methods also represent an ethical codex that is independent of culture, legislation and religion, the groups targeted by the 3Rs educational approach should span all relevant societal segments. The aim is to counter prejudice against 3Rs approaches by providing evidence of the scientific relevance and validity of 3 Rs methods.

\section{Dissemination channels}

As the target audience groups have different information requirements, well-established face-to-face teaching in classrooms and hands-on teaching in laboratories but also digital on-line courses, newspaper articles, TV and radio presentations, as well as blogs, meetings and expert reports were determined as worthwhile channels for communication and education (Fig. 1).

\section{Strategy of advertisement}

As "alternative methods" was considered an unclear term in a non-specialist context, it was suggested to announce courses under one of the following titles, as appropriate to the target audience: "Life science research in the $21^{\text {st }}$ century", "Modern approaches in the life sciences", "New methods in toxicology", "Theoretical base and methodical superstructure in modern life sciences", "The revolution of in vitro methods in life sciences".

Such strategic advertisement will draw attention to the topicality and complexity of 3Rs methodology and emphasize the dynamics of this rapidly developing scientific field.

\section{Modular structure of the educational program}

The complexity of the diverse 3Rs approaches together with the broad range of application fields and different demands of different audiences make the modular concept of the 3Rs education approach inevitable.

The lectures on education worked out by the expert panel will provide an integrated structure, which can be organized as modules addressing different aspects of 3 Rs methods. The depth of detailed content of each module can be adjusted to the requirements regarding the educational and scientific background and interests of the different audiences. This modular education system also allows providing each module as a stand-alone lecture on the professional level.

This modular system (Fig. 2) allows combining theoretical with practical (hands-on) aspects ranging in time span from a few hours to several days. The modules can be complemented with case stories, incorporation of study assignments, and hands-on work.

\section{Overview of teaching module content}

The following sections reflect the theoretical modules of the framework program worked out during the workshop (Fig. 2).

\subsection{Animals in science and society}

This module can be regarded as a general introduction to the course.

Animals are used by humans for a variety of purposes. While the use of animals as companion animals in general is perceived positively by society, other contexts of use, e.g., in the food industry, for entertainment purposes, as work animals and as laboratory animals, are seen more ambivalently. Understanding the different societal perceptions of the use and role of animals in science and society helps understand the origins of one's perceptions and emotional responses and to develop an attitude towards (laboratory) animals that is based on respect and their intrinsic value.

The debate on animal experimentation covering the biomedical benefits, advantages and disadvantages of animal models, basics of animal husbandry, animal laboratory sciences, animal behavior and stress responses should be covered here. Also, the interaction between science and society and the role of societal concerns regarding animal experimentation should be addressed (Kretlow et al., 2010).

The above mentioned aspects can be integrated to offer a framework for ethical considerations on the use of animals; the basic principles of ethics with the relevant decision-making tools, e.g., "Balance of Value against Costs" should be discussed (Festing and Wilkinson, 2007).

This module will generate awareness for identification of moral values from different point of views. For instance, what is the moral value of harming a volunteer in a clinical trial? What if he has signed a consent form? If the benefit is the same, can we take an animal for the same test instead of a person, though the animal cannot understand and sign any consent forms and he is not part of the trial by free will?

\subsection{Drivers for change towards 3Rs methods}

There are several major drivers for change towards 3Rs methods: scientific developments, societal changes, and economic aspects.

\section{Scientific developments}

The scientific limitations and problems with respect to extrapolation of results from animal models to the human situation will be discussed and described along with a variety of examples. 
Fig. 2: Overview of the modules of the education courses

Bold letters mark the modules covering different aspects of $3 R$ s methods, subchapters refer to the content of the teaching modules.

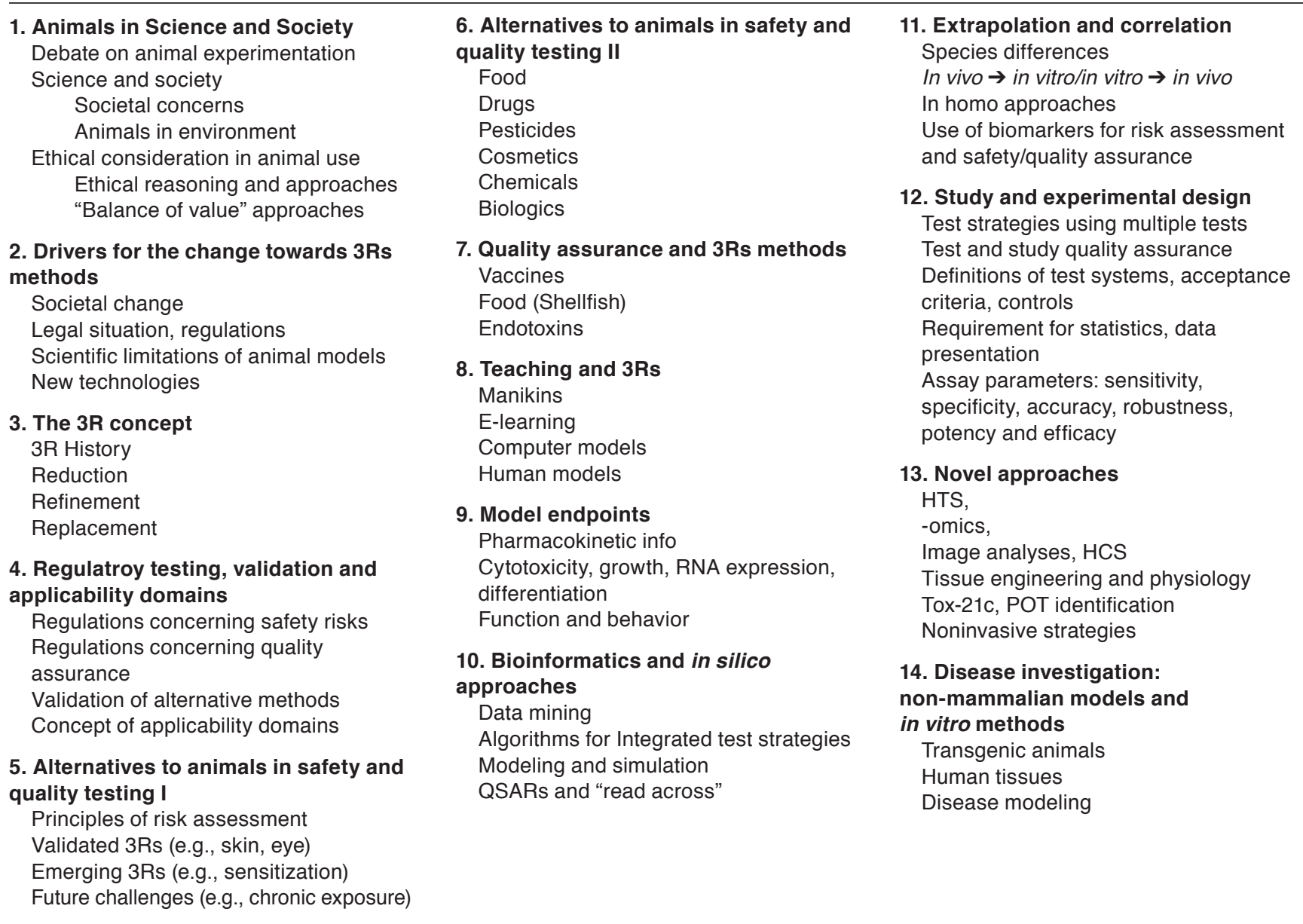

Also, the technological innovations such as high-content image analyzers, lab-on-chip and high-throughput approaches inter alia that now allow us to address scientific questions that cannot be adequately addressed with in vivo methods, e.g., toxicological effects of compound mixtures, will be discussed as drivers towards 3 Rs methods.

\section{Societal changes}

The milestones in societal changes and the associated revisions of regulations regarding the use of animals for research and testing purposes, i.e., introduction of animal tests, national animal protection law, multinational animal protection law, and differences in international animal protection laws as well as the introduction of international test guidelines for approved in vitro methodologies by the Organisation for Economic Co-operation and Development (OECD) and the International Conference on Harmonization shall be discussed. These developments have led to the current "No, unless... Principle."

\section{Economic aspects}

Increasingly, economic considerations such as duration (time to market), through-put (as an obstacle for frontloading toxicology in the drug development process) and costs for large-scale programs like REACH are becoming important drivers. In addition, the potential consequences of wrong business decisions, e.g., made based on extrapolation of animal data to humans, need to be stressed.

\subsection{The 3Rs concept}

In 1959 Russell and Burch published the important and now classic book, "The Principles of Humane Experimental Technique." In this book they defined humane science with the concept of the 3Rs - replacement, reduction and refinement. Replacement is the concept of using in vitro and other non-whole animal techniques to address a scientific question. Reduction expresses the concept of using the lowest number of animals without compromising scientific soundness and addresses the issue of experimental design. Refinement refers to using techniques and approaches that eliminate or decrease pain and distress experienced by the experimental animals.

An equally important contribution of Russell and Burch is their deduction that good science and animal welfare go hand in hand. This concept is now more generally accepted and is a driving force for appropriate experimental design .

Today the ideas from "The Principles of Humane Experimental Technique" have been incorporated into national and interna- 
tional regulations . They are endorsed by organizations around the world, and have a vast economic impact .

Russell and Burch changed the debate on the use of animals from a philosophical matter to a scientific issue. By introducing the principles of refinement, reduction and replacement (3Rs) as goals and guidelines for the work with animal subjects they put a high value on the integrity of living beings in scientific practice.

\subsection{Regulatory testing, validation and applicability domains}

Most regulatory authorities today rely on well-established animal-based models and test systems to assess the safety and efficacy of consumer products and chemicals, e.g., industrial chemicals, pesticides, cosmetics and pharmaceuticals. Animal testing is performed to determine whether and to what extent exposure to these compounds poses a risk to humans or to the environment.

\section{Regulatory testing}

The extent of regulatory testing will be increasing in the coming years as various national and international programs seek to obtain increasing amounts of toxicity information in the face of increasing public concern about the safety of chemicals. For example, large-scale programs for industrial chemicals are the REACH legislation (Registration, Evaluation, and Authorization and Restriction of Chemicals) in Europe and a possible TSCA (Toxic Substance Control Act) reauthorization under discussion in the US (Locke and Myers, 2011). Programs of this type could require huge numbers of animals if current guidance for testing is followed, however, language either exists (REACH Directive), or could be added (TSCA reauthorization), to support the use of non-animal methods wherever possible. Other examples for animal use intensive programs are the US endocrine disruptor screening program and its possible European counterpart. At this moment it is not clear, whether also the increasing concern about nanoparticle toxicity will lead to major testing demands (Silbergeld et al., 2011).

\section{Validation}

Before non-animal methods are incorporated into the regulatory standards of most countries they must be "validated" - the process of demonstrating that their use provides equivalent protection to that obtained from animal tests. In order to show that alternative methods can be at least as sensitive, reproducible, and relevant to predicting human health effects as the standard animal methods, national and supranational institutions, i.e., ICCVAM ${ }^{2}$, ECVAM $^{3}, \mathrm{JaCVAM}^{4}$, OECD ${ }^{5}$, and more recently KoCVAM in Korea and BraCVAM in Brazil, have been established for validation of alternative methods. Such institutions also function for the purpose of facilitating the implementation of these methods into regulatory testing, which is governed by national and supranational pharmacopoeias, e.g., the European Pharmacopoeia pub-

\footnotetext{
2 http://iccvam.niehs.nih.gov/

3 http://ecvam.jrc.it/

4 http://jacvam.jp/

5 http://www.oecd.org/

6 www.edqm.eu

7 http://www.usp.org/

8 http://www.nc3rs.org.uk/page.asp?id=1357
}

lished by EDQM ${ }^{6}$ and the US Pharmacopeia ${ }^{7}$. One key message is that validation is not a process set in stone, but one which needs to be adaptable to different needs and purposes .

Product and chemical risk assessment today uses non-animal in vitro tools more and more routinely. For example, advanced cell culture techniques aiming at reflecting absorption (uptake) and chemical metabolism are often used. To support these strategies, quality assurance programs involving "good practice" guidelines (Good Laboratory Practice, GLP; Good Manufacturing Practice GMP; Good Cell Culture Practice GCCP ) have been implemented. Additional guidelines, e.g., the ARRIVE guidelines ${ }^{8}$ on reporting of in vivo experiments or guidelines on study design and statistical analysis, ensure that the new toxicology methods will provide consistent and reliable results .

This module aims to inform interested parties (including regulators) about the regulatory status quo, the use of 3Rs in risk assessment, ITS (integrated/intelligent testing strategies), the role of validation in the acceptance and implementation process of new models and also about new concepts in the area of validation, e.g., the modular approach to validation (Hartung et al., 2004), WoE (weight of evidence validation), choice of points of reference, the concept of defining applicability domains for validated assays or the application of principles of "evidencebased medicine" .

\section{Applicability domains}

Not every chemical can be tested in every test; some types of chemicals are known to result in false positive or false negative test results. The concept of applicability domains is adapted from in silico approaches, and defines chemical classes and/or ranges of test method endpoints for which the model makes reliable predictions. The concept of applicability domains describes the fact that an alternative method may be proven valid only for a distinct range of compounds, e.g., the cytosensor microphysiometer test method is considered to be scientifically valid to identify mild ocular irritants only for water-soluble detergents . This new concept of applicability domains allows designing a battery of alternative test systems or a tiered testing strategy covering larger parts of the chemical universe. The applicability domain may also be broadened later by testing a broader range of substances and providing additional evidence of valid results.

\subsection{Alternative approaches to animals in safety and quality testing I}

3Rs testing strategies represent an opportunity not only to spare animals and limit their suffering, but also to develop human cell and tissue based test systems that more accurately predict adverse effects in humans. At the same time many of these new test systems should be amenable to use in high-throughput modes, which will allow the evaluation of a much larger number of compounds than the time-consuming traditional animal tests. Finally, since 
most in vitro tests are easier to interpret than a whole organism, it should be possible to identify directly the cellular mechanisms underlying the toxic effects by these strategies.

This is in line with the current shift in paradigm, from relying on apical endpoints in in vivo animal studies to a more basic understanding of adverse effects or Pathways of Toxicology (PoT) in a systems-biology approach (NRC, 2007; . With the maturation of mechanistic high-throughput approaches 3Rs methods have opened to new concepts in risk assessment, moving from animal-relevant in vivo methods to human-relevant multi-endpoint assays. The concept has been recently expanded to systematic mapping of pathways of toxicity (PoT), forming the human toxome .

Currently there are validated 3Rs methods, e.g., for skin and eye irritation or for phototoxic compounds, which could in part already be converted to automated high-throughput technologies. Moreover, there are also 3Rs methods in the pipeline, which are very promising, e.g., for evaluation of skin sensitizing compounds.

Future challenges that have to be met in the field of 3Rs methods include addressing the chronic exposure effects of substances. In this regard, reproductive toxicity, repeated dose toxicity, toxicokinetics and carcinogenicity are the fields, where nonanimal approaches have to be optimized and validated (Adler et al., 2011; Hartung et al., 2011).

The various approaches should be discussed in the module, which will include pathway of toxicity (PoT) based approaches and Integrated Testing Strategies (ITS), which are necessary, for example, to provide the detailed toxicological information that is required by the European REACH program (Rovida and Hartung, 2009). This module should discuss the principal advantages and limitations of the different approaches. A method critical approach should be applied not only to animal testing but also to its alternatives. In silico approaches, which can form an important part of any ITS, are discussed in Section 4.10.

\subsection{Alternative approaches to animals in safety and quality testing II}

3Rs approaches to animal use in safety testing and quality assurance are to be dealt with in correspondence with different application areas, e.g., food, drugs, pesticides, cosmetics, chemicals, biologics as well as pyrogenic or toxic contaminations. Here different application areas have different demands, different thresholds and thus their own concepts for safety testing.

- Food: Food safety problems are often related to microbial contamination, biotoxins (e.g. from algae in shellfish), allergens and chemical contaminations as well as food additives and contact materials.

- Drugs: Though there are many animal tests in pharmaceutical industry, the use of in vitro methods in pharmacology is also extensive, e.g., for high-throughput screening, for mechanistic studies, for metabolite profiling (P450 from liver microsomes), for genotoxicity (microsomes from aroclortreated animals) and for cytotoxicity testing.

- Pesticides: The US EPA defines pesticides as substances (also mixtures) repelling, controlling, preventing or destroying living organisms regarded as "pests", i.e., herbicides, insecticides, rodenticides, avicides, nematocides, antimicrobials, pheromones and genetically engineered toxins. Here alternative skin and eye irritation tests are promising replacement methods. Reduction and refinement alternatives such as tiered acute toxicity tests, the local lymphnode assay and the extended one-generation study for reproductive toxicity, play an enormous role due to the use of up to 6,000 animals per substance. Alternatives for ecotoxicological tests include the zebrafish egg test and the threshold approach for acute fish toxicity testing.

- Cosmetics: Relevant for the field of cosmetics there are significant achievements regarding validation and acceptance of alternative methods, e.g., for skin corrosion, phototoxicity, skin absorption, skin penetration, skin irritation, eye irritation, genotoxicity, mutagenicity and in part acute toxicity. On the other hand there are other alternative methods relevant for testing of cosmetics, which have to be optimized and validated: a) skin sensitization, b) toxicokinetics (especially for in vitro to in vivo extrapolation), c) carcinogenicity testing, especially the completion of the cell transformation assay validation and novel mutagenicity tests due to the high falsepositive rate of the existing ones, d) for reproductive toxicity, which is not mimicked completely in vitro by the battery originating from the ReProTect project and e) for repeated dose toxicity in vitro, which cannot be modeled even with complex in vitro systems and thus requires novel concepts such as PoT-based high-throughput testing and systems toxicology (Adler et al., 2011; Hartung et al., 2011).

- Chemicals: Notably, there are over 70 million chemicals, which have been synthesized to date. It is estimated that today there are about 100,000 synthetic chemicals consumers in Europe and US are exposed to, but there are safety assessments only for $5 \%$ of these substances. If the possible synergistic effects of mixtures or the various formulations of a given substance in nanoparticles should also be determined, there is a need for a tremendous amount of testing in the area of chemical safety .

- Biologicals: Biologicals (medicinal preparations made from living organisms and their products, including serums, vaccines, antigens, antitoxins, etc.) are often produced in bacteria or human cells and therefore a variety of special problems is associated with them. Special ways to detect culture contaminants have to be included in each production step and the problem of batch-to-batch variation has to be addressed carefully. Each batch might be unique and has to be tested extensively for safety, quality and efficacy. The regulation and legislation for biologicals is rather complex and in some cases they fall under different laws. Major progress has been made for the safety and efficacy testing of some vaccines and the determination of pyrogenic contaminations (see next section). Another, most prominent, recent example is the testing of Botox, the second most used cosmetic in 2001 (according to the International Society of Aesthetic Plastic Surgeons), which so far required titration of the potency of each batch in a mouse $\mathrm{LD}_{50}$ acute toxicity test, and is therefore one of the biggest animal consumers worldwide. It is regulated as a drug for relatively rare clinical conditions (though this might 
change as FDA approved Botox in 2010 for migraine), but more than $90 \%$ of all produced Botox is currently used for cosmetic purposes. Noteworthy, the first alternative test has recently (June 2011) been approved by FDA for Allergan, a major Botox producer.

\subsection{Quality assurance and 3Rs methods}

Quality assurance, safety control and animal testing are traditionally closely linked.

This module will deal with the difficulties and also success stories of exemplary tests on vaccines, marine food and endotoxin contamination (Box 2).

\section{Vaccines}

The US National Center for Immunization and Respiratory Diseases states: "For each birth cohort vaccinated with seven vaccines, direct health care costs are reduced by $\$ 9.9$ billion, 33,000 lives are saved, and 14 million cases of disease are prevented". Safety and potency of vaccines have to be proven extensively for every batch of the traditionally produced vaccines (in contrast to those produced by gene technology) in animal experiments associated with a significant level of pain and distress. Some examples of recent achievements in the quality control of toxoid vaccines are the replacement of challenge procedures by serological methods, the reduction of numbers of animals required by changing from multi-dose to single dose testing, and developments in the area of in vitro models and physicochemical techniques (Hendriksen, 2006).

\section{Marine food}

Because food safety is a matter of sensible importance, there are lower detection levels for food contaminants, strict standards, quality controls and monitoring procedures for food, which lead to a relatively high number of food safety alerts in marine products. The rigorous safety attempts in this field costs in Europe 400,000 lives of laboratory mice each year alone for the mouse bio-assay for accumulated algae toxins in shellfish. An LC-MS/ MS replacement for the mouse bioassay for the detection of Diarrhetic Shellfish Poisoning marine biotoxins was recently approved in Europe ${ }^{9}$.

\section{Endotoxin contamination}

Pyrogenic (fever-inducing) contamination in pharmaceuticals is a serious health problem. This contamination can be due to microbial (Gram-negatives, Gram-positives, fungi) organisms and their fragments as well as caused by particulate organic and inorganic matter. The Rabbit pyrogen test consumes more than 100,000 rabbits per year in Europe, and reflects the human reactivity only partially. This still high animal use continues despite the fact that to a large part it was replaced by the non-validated Limulus amebocyte lysate (LAL or Bacterial Endotoxin Test, BET) test. The LAL detects only pyrogens derived from Gramnegative bacteria. The human whole blood assay and some other monocyte activation assays (MAT) were adapted recently, after validation for detection of pyrogens in parenteral drugs. This test represents a functional and physiological assay that has been added to the European Pharmacopoeia as a replacement for the in vivo assay.

\subsection{Teaching and 3 Rs}

In 2008, 1.7\% (approximately 200,000) of the animals used for experimental and scientific purposes in the EU were used for education and training (Sixth Report on the Statistics on the Number of Animals used for Experimental and other Scientific Purposes in the Member States of the European Union SEC(2010) $1107^{10}$. Animals euthanized before scientific proce-

Box 2

For course discussions it may be helpful to present examples for advantages and limitations of 3 Rs models as below:

\section{Success of Replacement}

Monoclonal antibodies (ascites); Calcitonin batch control (Novartis); Phototoxicity (OECD guidelines); Pyrogen Test; Botox batch control; Goldorfen (Leuciscus idus) test for water purity in Germany (DIN 38412 L31); Skin corrosion; Skin irritation, Eye irritation, Genotoxicity

\section{Additional, or faster, or cheaper information compared to traditional approaches}

Cell culture for pharmacological screening; LLNA for sensitization; New LD $_{50}$ test approaches; Ecotoxicology acute toxicity testing (daphnia, fish, algae); Non-invasive imaging; Vaccine potency testing

\section{Failure of animal approaches}

Thalidomide; Delayed organophosphate-induced neuropathy in the rat; Biologicals testing (humanized antibodies, therapeutically used cytokines); Neurodegenerative disease modeling

\section{Species non-correlation}

MPTP in rat vs. mouse; vomiting response in rodents, Biologicals and immunotherapeutics testing

\section{Animals still required}

Repeat dose toxicity, carcinogenicity of drugs, behavioral pharmacology, unknown marine biotoxins, biocompatibility of medical devices, cardiovascular regulation

\footnotetext{
9 http://eur-lex.europa.eu/LexUriServ/LexUriServ.do?uri=OJ:L:2011:006:0003:0006:EN:PDF

$10 \mathrm{http}: / /$ eur-lex.europa.eu/LexUriServ/LexUriServ.do?uri=COM:2010:0511:REV1:EN:PDF
} 
dures are not considered in these statistics; still, they are used in large numbers for dissection and anatomy classes. Live animals are used, e.g., to teach experimental procedures and laboratory skills for graduate students in disciplines like anatomy, physiology, biochemistry, pharmacology, psychology, parasitology, surgery and toxicology .

In recent years many non-harmful alternatives have been made available for teaching purposes :

- (3D-) models, mannequins and mechanistic simulators

- Film and interactive video

- Computer simulation and virtual reality

- Non-invasive self-experimentation and human studies

- Plant experiments

- Observational and field studies

- Materials from slaughterhouses and fisheries

- Permanently preserved specimens

- In vitro studies in cell lines

- Animal cadavers from an animal-friendly and ethical source such as from animals euthanized for medical reasons

- Supervised clinical experience

Teaching with non-animal educational models and the outcome of the learning objectives have been evaluated scientifically, though definitive studies are rare. In the most cases animal-free teaching attained similar or better results . Lack of this knowledge and reluctance of some teachers are still major hurdles for the introduction of 3Rs methods in education (Schmidt et al., 2011). The role of animals and new animal-free methods should be discussed as well as approaches that limit animal use in skills training.

\subsection{Model endpoints}

Particularly in the field of toxicological risk assessment, information concerning human safety carried out in animal models is limited by the need to extrapolate data from the animal to the human system . There is a need to develop endpoints for 3Rs methods mirroring toxic effects in man. Moreover, for the improvement of the predictive properties of 3 Rs methods, testing of a large number of compounds with these methods will be required. Since the molecular targets of toxicity are not known for new substances, a battery of endpoints, such as receptor and enzyme targets, must be combined in the assessment procedure. How primary cell reactions, such as those of glia (Falsig et al., 2004a,b, 2006), can be related to in vivo data (Lund, 2006), and then on this basis translated to cell lines (Henn et al., 2009) will explain this concept using a recent example.

This education module will provide strategies to identify relevant endpoints for the evaluation of defined problems with 3Rs methods. It will be necessary to consider, among others, kinetic background information, involving data on cytotoxicity, growth, RNA expression, and differentiation, and functionbehavior relationships.

\subsection{Bioinformatics and in silico approaches}

A number of in silico tools that contribute to replacing and refining animal testing have entered toxicology (rule-based models, (quantitative) structure activity relationships, virtual organs, kinetic modeling, systems biology, etc.) . The data analysis procedures (DAP) for these methodologies often require calculations and statistical analysis of the results in silico. Statistical evaluation of data or methods can lead to reducing group sizes or abandoning tests shown to be irrelevant.

Many 3Rs methods create computational needs: New 3Rs approaches often need tools for analyzing large amounts of data. Such data usually result from multi-endpoint approaches (omics, image analysis technologies), or high-throughput testing (HTS). Stand-alone "non-testing" alternative methods include (quantitative) structure activity relationships ((Q)SAR), rule-based systems, read-across, etc. The field of computational toxicology also includes modeling and simulation, e.g., physiology-based pharmaco-(bio-)kinetic modeling (PBPK) or receptor binding models. All of these aspects have major in silico components.

Various approaches and tools are frequently involved in the analysis of data created with 3Rs methods, e.g., data mining, integrated test strategies (ITS) and computational modeling . The educational module concerning bioinformatics and in silico methods will inform and train interested parties in handling, analyzing, and interpreting data and information collected with these approaches.

\subsection{Extrapolation and correlation}

Data collected with 3Rs methods are still subjected to comparative studies with in vivo data to prove their validity or in proof of principle studies. Here the concept and application of the in vitroin vivo correlation (IVIVC) draws the attention of pharmaceutical and chemical industry, but also of academia and regulatory players. However, species differences in uptake, absorption, metabolism, kinetics and excretion pose major obstacles for interpretation of IVIVC evaluation, which can sometimes be overcome if the modeling and the parameterization are human-based.

The most relevant systems are in homo approaches, such as microdosage application in development and optimization of formulation of therapeutic agent.

This module will teach IVIVC and differences of species with regard to biokinetics and toxicodynamics. Assessment of the value of in homo approaches as well as their explanatory power and limitations for different sectors also will be addressed. The involvement of biomarkers for risk assessment, safety and quality assurance using IVIVC will be included in this module.

\subsection{Study and experimental design}

The quality of study and experimental design is essential for collecting relevant data; generation of a series of experiments based on phenotypically minor variations to refine previous work is a negative example of scientific study and experimental design.

In case of animal studies the choice of species will be discussed as well the role of inbred and outbred animals with respect to reducing animal numbers and increasing scientific validity.

The expert panel recommended for this module to provide definitions for test systems, mediate scientific and regulatory acceptance criteria and define the necessary strategy for collecting adequate controls. Also the statistical background shall be taught to ensure that the requirements, e.g., sufficient replicates for the appropriate statistical analysis, are met. Also the assay param- 
eters sensitivity, specificity, accuracy, robustness, potency, efficacy will be introduced using examples of tests and studies (Leist et al., 2010; Zimmer et al., 2011; Stiegler et al., 2011).

\subsection{Novel approaches}

Robotics, liquid handling devices, sensitive detectors and control software resulting in automated experimentations and data processing enable the creation of millions of data points within a short time. The educational module for novel approaches will provide knowledge on the latest technological developments relevant to the $3 \mathrm{R}$ s field. At present, this module shall provide insight into the field of high-throughput systems (HTS), -omics (proteomics, transcriptomics, metabolomics), image analysis with high-content systems, tissue engineering and physiology, and discuss strategies for identification of pathways of toxicity (PoT).

In this module also current groundbreaking concepts will be addressed, e.g., the idea of Tox-21c (NRC, 2007): this report illuminates how the explanatory power of toxicological risk assessment can be maximized by employing high-throughput in vitro screening assays, tests in lower organisms, systems biology, functional genomics and transcriptomics as well as predictive in silico approaches.

\subsection{Disease investigation: non-mammalian models and in vitro methods}

It is essential to keep in mind that biomedical research is largely focused on the understanding of human diseases. Despite the interspecies differences reflected inter alia in anatomical, biochemical and life span variations, animal models are still widely studied models in biomedical research focusing on human diseases.

The most important tool in the area of disease modeling leading to strong increases in animal use are transgenic animals, which are created and used as disease models. One aim of this course will be to delineate the limitations of and alternatives to the use of mammalian models. First we will cover the use of non-mammalian models. Second we will study the rapid development of in vitro methods .

\section{Non-mammalian models}

Although transgenic mice (and more recently rats) are heralded as the gold standard of genetic research, many alternative models have been used with extraordinary success, ranging from fish eggs (especially from zebrafish), insects (Drosophila), helminthes (C. elegans) and plants to unicellular organisms (yeast, dictyostelium). The course will detail a few examples where these alternative models have proven particularly successful, and compare the advantages and limitations of various models.

\section{In vitro technologies}

These technologies can be classified into two categories: human and non-human cells in vitro. Novel techniques providing human tissues in vitro or ex vivo already showed a promising potential to reduce animal use and in some cases to replace the animal model. Especially the field of (human) embryonic and induced pluripotent stem cells (iPSC) is receiving large atten- tion. The generation of cells and tissues with patient-specific background is seen as an important alternative to the use of animal models.

More generally, as our knowledge progresses, more in vitro methods are being developed. One of the most striking examples is the development of recombinant antibody technologies which allow selection and production of antibodies in vitro.

\section{Information and resources}

The expert panel of this workshop suggested providing a public online repository with presentations also containing comments of authors regarding the modules discussed in this report. Also a web-based information pool should be created for related and relevant information, e.g., case reports on 3Rs approaches. This should complement more specialized information provided in articles, books and journals.

\section{Conclusions and recommendations}

The workshop mapped the full bandwidth of 3Rs approaches in biomedical research, toxicology and the life sciences. A variety of target groups could benefit from the offers of such a comprehensive curriculum. Virtual education offers should help to make contents more broadly available. In order to expand the number of teachers, "train-the-trainer" approaches and support by sharing content and materials for education were considered most helpful.

By defining a non-exhaustive list of modules to be considered, a step toward such a curriculum was done. It was recommended to carry out practical trials and document them properly to further define a common curriculum (and its adaption to different areas and target groups). CAAT-Europe is prepared to coordinate these activities, and to offer the different modules to interested parties.

\section{References}

Adler, S., Basketter, D., Creton, S., et al. (2011). Alternative (nonanimal) methods for cosmetics testing: current status and future prospects - 2010. Arch. Toxicol. 85, 367-485.

Bahramsoltani, M., Plendl, J., Janczyk, P., et al. (2009). Quantitation of angiogenesis and antiangiogenesis in vivo, ex vivo and in vitro - an overview. ALTEX 26, 95-107.

Balls, M., Amcoff, P., Bremer, S., et al. (2006). The principles of weight of evidence validation of test methods and testing strategies. The report and recommendations of ECVAM workshop 58. ATLA 34, 603-620.

Bauer, M. S. (1993). A survey of the use of live animals, cadavers, inanimate models, and computers in teaching veterinary surgery. J. Am. Vet.Med.Assoc. 203, 1047-1051.

Boekelheide, K. and Andersen, M. E. (2010). A mechanistic redefinition of adverse effects - a key step in the toxicity testing paradigm shift. ALTEX 27, 243-252.

Bottini, A. A., Amcoff, P., and Hartung, T. (2007). Food for thought 
... on globalisation of alternative methods. ALTEX 24, 255-269.

Bottini, A. A. and Hartung, T. (2009). Food for thought ... on the economics of animal testing. ALTEX 26, 3-16.

Brunner, D., Frank, J., Appl, H., et al. (2010). Serum-free cell culture: the serum-free media interactive online database. ALTEX 27, 53-62.

Coecke, S., Balls, M., Bowe, G., et al. (2005). Guidance on good cell culture practice. a report of the second ECVAM task force on good cell culture practice. ATLA 33, 261-287.

Daneshian, M., Leist, M., and Hartung, T. (2010). The Center for Alternatives to Animal Testing - Europe (CAAT-EU): a transatlantic bridge for the paradigm shift in toxicology. ALTEX 27, 63-69.

Dietrich, D. R. (2010). Courage for simplification and imperfection in the $21^{\text {st }}$ century assessment of "Endocrine disruption". ALTEX 27, 264-278.

Ducceschi, L., Green, N., and Miller-Spiegel, C. (2010). Dying to learn: the supply and use of companion animals in U.S. colleges and universities. ALTEX 27, 304-308.

Falsig, J., Latta, M., and Leist, M. (2004a). Defined inflammatory states in astrocyte cultures: correlation with susceptibility towards CD95-driven apoptosis. J. Neurochem. 88, 181-193.

Falsig, J., Pörzgen, P., Lotharius, J., and Leist, M. (2004b). Specific modulation of astrocyte inflammation by inhibition of mixed lineage kinases with CEP-1347. J. Immunol. 173, 2762-2770.

Falsig, J., Pörzgen, P., Lund, S., et al. (2006). The inflammatory transcriptome of reactive murine astrocytes and implications for their innate immune function. J. Neurochem. 96, 893-907.

Festing, S. and Wilkinson, R. (2007). The ethics of animal research. Talking Point on the use of animals in scientific research. EMBO Rep. 8, 526-530.

Gruber, F. P. and Dewhurst, D. G. (2004). Alternatives to animal experimentation in biomedical education. ALTEX 21, Suppl. 1, 33-48.

Hagelschuer, I., Hauff, P., Strube, A., et al. (2009). Luminescent imaging technology as an opportunity to reduce and refine animal experiments: Light at the end of the tunnel? ALTEX 26, 177-185.

Hamann, M., Lange, N., Kuschka, J., et al. (2010). Non-invasive genotyping of transgenic mice: comparison of different commercial kits and required amounts. ALTEX 27, 185-190.

Hart, L. A., Wood, M. W., and Weng, H. Y. (2005). Mainstreaming alternatives in veterinary medical education: resource development and curricular reform. J. Vet. Med.Educ. 32, 473-480.

Hartung, T., Bremer, S., Casati, S., et al. (2004). A modular approach to the ECVAM principles on test validity. ATLA 32, 467-472.

Hartung, T. (2007). Food for thought ... on validation. ALTEX 24, 67-80.

Hartung, T. and Leist, M. (2008). Food for thought ... on the evolution of toxicology and the phasing out of animal testing. ALTEX 25, 91-102.

Hartung, T., Blaauboer, B., and Leist, M. (2009). Food for thought ... on education in alternative methods in toxicology. ALTEX 26, 255-263.

Hartung, T. and Hoffmann, S. (2009). Food for thought ... on in silico methods in toxicology. ALTEX 26, 155-166.

Hartung, T. and Rovida, C. (2009). Chemical regulators have over- reached. Nature 460, 1080-1081.

Hartung, T. (2010a). Food for thought ... on alternative methods for chemical safety testing. ALTEX 27, 3-14.

Hartung, T. (2010b). Comparative analysis of the revised Directive 2010/63/EU for the protection of laboratory animals with its predecessor 86/609/EEC - a t ${ }^{4}$ report. ALTEX 27, 285-303.

Hartung, T. (2010c). Evidence-based toxicology - the toolbox of validation for the $21^{\text {st }}$ century? ALTEX 27, 253-263.

Hartung, T. (2010d). Food for thought ... on alternative methods for nanoparticle safety testing. ALTEX 27, 87-95.

Hartung, T., Bruner, L., Curren, R., et al. (2010). First alternative method validated by a retrospective weight-of-evidence approach to replace the Draize eye test for the identification of non-irritant substances for a defined applicability domain. ALTEX 27, 43-51.

Hartung, T. and McBride, M. (2011). Food for thought ... on mapping the human toxome. ALTEX 28, 83-93.

Hartung, T., Blaauboer, B. J., and Bosgra, S. (2011). An expert consortium review of the EC-commissioned report "Alternative (Non-Animal) Methods for Cosmetics Testing: Current Status and Future Prospects - 2010". ALTEX 28, 183-209.

Hendriksen, C. F. (2006). Towards eliminating the use of animals for regulatory required vaccine quality control. ALTEX 23, 187-190.

Henn, A., Lund, S., Hedtjarn, M., et al. (2009). The suitability of BV2 cells as alternative model system for primary microglia cultures or for animal experiments examining brain inflammation. ALTEX 26, 83-94.

Hoffmann, S., Peterbauer, A., Schindler, S., et al. (2005). International validation of novel pyrogen tests based on human monocytoid cells. J. Immunol. Methods 298, 161-173.

Hoffmann, S., Edler, L., Gardner, I., et al. (2008). Points of reference in the validation process: the report and recommendations of ECVAM Workshop 66. ATLA 36, 343-352.

Jaworska, J. and Hoffmann, S. (2010). Integrated Testing Strategy (ITS) - Opportunities to better use existing data and guide future testing in toxicology. ALTEX 27, 231-242.

Jukes, N. and Chiuia, M. (2003). From guinea pig to computer Mouse: Alternative methods for progressive human Education. Leicester: InterNICHE

Kamusella, P. C., Wissgott, C., Grosse-Siestrup, C., et al. (2009). A model of isolated, autologously hemoperfused porcine slaughterhouse lungs. ALTEX 26, 279-284.

Karleta, V., Andrlik, I., Braunmuller, S., et al. (2010). Poloxamer 188 supplemented culture medium increases the vitality of Caco-2 cells after subcultivation and freeze/thaw cycles. ALTEX 27 , 191-197.

Knight, A. (2007). The effectiveness of humane teaching methods in veterinary education. ALTEX 24, 91-109.

Knight, A. (2008). Non-animal methodologies within biomedical research and toxicity testing. ALTEX 25, 213-231.

Kohler, G. and Milstein, C. (2005). Continuous cultures of fused cells secreting antibody of predefined specificity. 1975. J. Immunol. 174, 2453-2455.

Kretlow, A., Butzke, D., Goetz, M. E., et al. (2010). Implementation and enforcement of the 3Rs principle in the field of transgenic animals used for scientific purposes. Report and recommendations of the BfR expert workshop, May 18-20, 2009, Berlin, Germany. ALTEX 27, 117-134. 
Krewski, D., Acosta, D., Jr, Andersen, M., et al. (2010). Toxicity testing in the 21 st century: a vision and a strategy. J. Toxicol. Environ. Health B Crit. Rev. 13, 51-138.

Leist, M., Bremer, S., Brundin, P., et al. (2008a). The biological and ethical basis of the use of human embryonic stem cells for in vitro test systems or cell therapy. ALTEX 25, 163-190.

Leist, M., Kadereit, S., and Schildknecht, S.(2008b). Food for thought ... on the real success of 3R approaches. ALTEX 25, 17-32.

Leist, M., Hartung, T., and Nicotera, P. (2008c). The dawning of a new age of toxicology. ALTEX 25, 103-114.

Leist, M., Efremova, L., and Karreman, C. (2010). Food for thought ... considerations and guidelines for basic test method descriptions in toxicology. ALTEX 27, 309-317.

Lima, W. C., Lelong, E., and Cosson, P. (2011). What can Dictyostelium bring to the study of Pseudomonas infections? Semin. Cell Dev. Biol. 22, 77-81.

Locke, P. and Myers D. B. (2011). A replacement-first approach to toxicity testing is necessary to successfully reauthorize TSCA. ALTEX 28, 266-272.

Lotharius, J., Falsig, J., van Beek, J., et al. (2005). Progressive degeneration of human mesencephalic neuron-derived cells triggered by dopamine-dependent oxidative stress is dependent on the mixed-lineage kinase pathway. J. Neurosci. 25, 6329-6342.

Lund, S., Christensen, K. V., Hedtjärn, M., et al. (2006). The dynamics of the LPS triggered inflammatory response of murine microglia under different culture and in vivo conditions. J. Neuroimmunol. 180, 71-87.

Manzer, A. K., Lombardi-Borgia, S., Schafer-Korting, M., et al. (2009). SV40-transformed human corneal keratocytes: optimisation of serum-free culture conditions. ALTEX 26, 33-39.

Martinsen, S. and Jukes, N. (2005). Towards a humane veterinary education. J. Vet. Med. Educ. 32, 454-460.

Maynard, J. and Georgiou, G. (2000). Antibody engineering. Annu. Rev. Biomed. Eng. 2, 339-376.

NRC (2007). Toxicity testing in the twenty-first century: A vision and a strategy. Washington, DC: National Academies Press.

Rovida, C. and Hartung, T. (2009). Re-evaluation of animal numbers and costs for in vivo tests to accomplish REACH legislation requirements for chemicals - a report by the transatlantic think tank for toxicology $\left(\mathrm{t}^{4}\right)$. ALTEX 26, 187-208.

Sauer, U. G. (2009a). Animal and non-animal experiments in nanotechnology - the results of a critical literature survey. ALTEX $26,109-128$.

Sauer, U. G. (2009b). Political incentives towards replacing animal testing in nanotechnology? ALTEX 26, 285-294.

Sauer, U. G., Wachter, T., Grune, B., et al. (2009). Go3R - semantic Internet search engine for alternative methods to animal testing. ALTEX 26, 17-31.

Schindler, S., Spreitzer, I., Loschner, B., et al. (2006). International validation of pyrogen tests based on cryopreserved human primary blood cells. J. Immunol. Methods 316, 42-51.

Schindler, S., von Aulock, S., Daneshian, M., et al. (2009). Development, validation and applications of the monocyte activation test for pyrogens based on human whole blood. ALTEX 26, 265277.

Schmidt, A., Hohensee, C., Teichgräber, U., and Schmidt,A. (2011). SATIS ethics ranking of universities in Germany regarding ani- mal use in education. ALTEX 28, 243-244.

Shoemaker, C. B. (2005). When will rAbs replace mAbs in labs? Vet.J. 170, 151-152.

Siegel, D. L. (2002). Recombinant monoclonal antibody technology. Transfus. Clin. Biol. 9, 15-22.

Silbergeld, E. K., Contreras, E. Q., Hartung, T., et al. (2011). Nanotoxicology: "The end of the beginning" - Signs on the roadmap to a strategy for assuring the safe application and use of nanomaterials. ALTEX 28, 236-241.

Skelin, M., Rupnik, M., and Cencic, A. (2010). Pancreatic beta cell lines and their applications in diabetes mellitus research. ALTEX $27,105-113$.

Stebbings, R., Findlay, L., Edwards, C., et al. (2007). "Cytokine storm" in the phase I trial of monoclonal antibody TGN1412: better understanding the causes to improve preclinical testing of immunotherapeutics. J. Immunol. 179, 3325-3331.

Stiegler, N. V., Krug, A. K., Matt, F., and Leist, M. (2011). Assessment of chemical-induced impairment of human neurite outgrowth by multiparametric live cell imaging in high-density cultures. Toxicol. Sci. 121, 73-87.

Stingl, L., Volkel, M., and Lindl, T. (2009). 20 Years of hypertension research using genetically modified animals: no clinically promising approaches in sight. ALTEX 26, 41-51.

Stokes, W. S. and Wind, M. (2010). Validation of innovative technologies and strategies for regulatory safety assessment methods: challenges and opportunities. ALTEX 27,87-95.

Ullrich, A., Stolz, D. B., Ellis, E. C., et al. (2009). Long term cultures of primary human hepatocytes as an alternative to drug testing in animals. ALTEX 26, 295-302.

van Ravenzwaay, B. (2010). Initiatives to decrease redundancy in animal testing of pesticides. ALTEX 27, 159-161.

van Vliet, E. (2011). Current standing and future prospects for the technologies proposed to transform toxicity testing in the $21^{\text {st }}$ century. ALTEX 28, 17-44.

Vedani, A., Smiesko, M., Spreafico, M., et al. (2009). VirtualTox$\mathrm{Lab}$ - in silico prediction of the toxic (endocrine-disrupting) potential of drugs, chemicals and natural products. Two years and 2,000 compounds of experience: a progress report. ALTEX 26, 167-176.

Vogel, R. (2009). Alternatives to the use of animals in safety testing as required by the EU-Cosmetics Directive 2009. ALTEX 26, 223-226.

Zimmer, B., Schildknecht, S., Kuegler, P. B., et al. (2011). Sensitivity of dopaminergic neuron differentiation from stem cells to chronic low-dose methylmercury exposure. Toxicol. Sci. 121, 357-367.

\section{Correspondence to}

Mardas Daneshian, PhD

University of Konstanz

POB 600

78457 Konstanz

Germany

Phone: +497531882233

Fax: +497531884156

e-mail: mardas.daneshian@uni-konstanz.de 